\title{
Parametric Study for the Estimation of Optimum Vortex Generators Arrangement in Straight Pipes
}

\author{
Katemis K. Ioannis, Douvi C. Eleni, Margaris P. Dionissios
}

\begin{abstract}
The objective of the present study is the parametric study of secondary geometries, in particular Wavy Fins, added in pipes in order to enhance heat transfer. Simulations carried out in ANSYS Fluent for different Wavy Fin arrangement and geometries for Reynolds numbers varying from 10,000 to 20,000 , using $k-\varepsilon$ turbulence model and wall functions. It is found that the mechanisms that enhance heat transfer caused by the fins are swirl flow and efficient mixing of the air, as well as boundary layer disturbance. Staggered arrangement found the most efficient in terms of heat transfer comparing to Discontinuous as well as the arrangement with $180^{\circ}$ phase shift. Assessing geometries concerning heat transfer Triangular Fin and Traditional Wavy Fin are very close, while Trapezoidal Fin has weaker heat transfer improvement. Results also show that adding protrusions also enhances heat transfer remarkably, while decreases pressure drop.
\end{abstract}

Index Terms - Wavy Fin, Heat Transfer, Vortex Generator, Computational Fluid Dynamics

\section{INTRODUCTION}

The plate fin heat exchanger (PFHE) is a compact heat exchanger widely used in cryogenic processes, aerospace, petrochemical and gas production and other industrial applications. PFHEs attractiveness lies in their compact structure, high efficiency, low cost, and ease of handling multiple streams [1]-[3].

Extended or finned surfaces such us louver fin, offset strip fin and wavy fin are widely used in PFHEs not only to enhance the heat transfer but reduce their size as well. Among these, wavy fins are particularly preferred for their simplicity of manufacture, potential for enhanced thermal-hydraulic performance [4]-[6].

A number of studies have begun to examine relation between Wavy Fin array characteristics and thermal-hydraulic performance. In 2006, Dong Junqi, Chen Jiangping, Chen Zhijiu, Zhou Yimin, Zhang Wenfeng [7] studied experimentally 11 heat exchangers having Wavy Fins. They conducted tests for various air side Reynolds numbers, ranging from 800 up to 6,500 with different fin dimensions and fin pitches. The characteristics of heat transfer and pressure drop for different geometry parameters were reported in terms of $\mathrm{j}$-factor and friction factor $\mathrm{f}$, as a

Ioannis K. Katemis, Department of Mechanical Engineering and Aeronautics, University of Patras, Patras, Greece

Eleni C. Douvi, Department of Mechanical Engineering and Aeronautics, University of Patras, Patras, Greece

Dionissios P. Margaris, Department of Mechanical Engineering and Aeronautics, University of Patras, Patras, Greece function of Re. Results show that both $\mathrm{j}$ and $\mathrm{f}$ factors increase with fin space increasing at the same $\mathrm{Re}$, the $\mathrm{j}$ factor increases with fin height, while the fin height has little effect on the $f$ factor as a function of Re.

In a follow-up study done in 2015, Yidan Song, Masoud Asadi, Gongnan Xie, L.A.O. Rocha [8] studied three dimensionless variables such as the channel space (S), the wavelength ratio $\left(\lambda_{1} / \lambda_{2}\right)$ and the amplitude ratio $\left(\alpha_{1} / \alpha_{2}\right)$ of two wavy walls to find the optimal configuration of wavy-fin. Two dimensional numerical simulations were conducted to obtain the steady laminar heat transfer and pressure drop characteristics by using computational fluid dynamics (CFD). According to the results, the optimal configuration lies in the range of $\mathrm{S}=0.13, \quad\left(\mathrm{a}_{1} / \mathrm{a}_{2}\right)=0.8$ and $0.8<\left(\lambda_{1} / \lambda_{2}\right)<0.9$. This optimal configuration results in increasing the rates of heat transfer by around $26 \%$ and decreasing the pressure drop by $54 \%$.

In a recent cross-sectional study on the previous year, Jian Wen, Ke Li, Chunlong Wang, Xing Zhang, Simin Wang [9] investigated the effects of inlet velocity and five configuration parameters (fin height, fin space, fin thickness, fin wavelength and double amplitude) on heat transfer and flow resistance of wavy fin array. The results agreed with previous studies that the $\mathrm{j}$ factor increases with the increase of fin space and fin height, and decreases with the increase of fin thickness, wavelength and inlet velocity. The $\mathrm{j}$ factor firstly increases with the increase of double amplitude and then decreases. The f factor increases with double amplitude, fin space and fin height, and decreases with fin thickness, wavelength and inlet velocity.

Over the past years, most research in wavy fins has emphasized in changes done to traditional wavy fin for further improvement of thermal-hydraulic characteristics. For instance, Yuan Xue, Zhihua Ge, Xiaoze Du and Lijun Yang (2018) [10] examined numerically three new types of wavy plate fins, namely perforated wavy fin, staggered wavy fin and discontinuous wavy fin in CFD simulations. The effects of key design parameters, including perforation diameters, staggered ratios and breaking distance were investigated, respectively, with Reynolds number changes from 500 to 4.500. It was found that swirl flow and efficient mixing of the fluid strengthened which leaded to heat transfer enhancement at all cases compared to the traditional wavy fin. At the same time, serration is beneficial to reduce the friction factor, and the breaking technique can reduce heat transfer area.

Similarly, Kitti Nilpueng, Ho Seon Ahn, Dong-Wook Jerng, Somchai Wongwises, [11] presented new experimental data on the influence of phase shift and air 
velocity on heat transfer coefficient, and pressure drop of airflow through wavy plate fin heat sinks. Wavy plate fins with a wavelength of $18.68 \mathrm{~mm}$, amplitude of $2.0 \mathrm{~mm}$, and phase shift of $0^{\circ}, 90^{\circ}$, and $180^{\circ}$ were used, while air velocity ranged between 1 and $5 \mathrm{~m} / \mathrm{s}$. The results showed that the higher phase shift and air velocity lead to the enhancement of the heat transfer coefficient and pressure drop. The heat transfer coefficient of the finned heat sink was enhanced when increasing the phase shift compared with a phase shift of $0^{\circ}$.

This study aims on the parametric study of wavy fins of seven different configurations, which enhance heat transfer when they are added in pipes. Various Reynolds numbers were examined in order to show the dependency of heat transfer from the air flow velocity.

\section{NUMERICAL ANALYSIS}

\section{A. Geometry}

The first step in the simulation procedure is the design of a rectangular pipe where the fins are located for each case studied. The pipe is represented in Fig.1 shown the entrance and exit length as well as the area where fins are located.

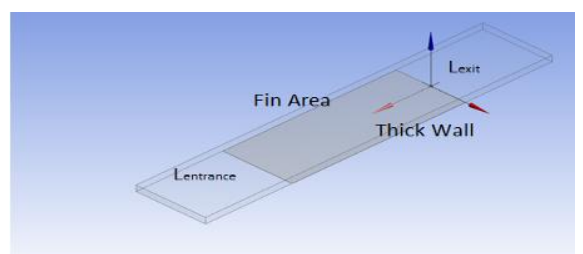

Fig. 1. Rectangular pipe geometry.

The entrance length is necessary to obtain a fully developed velocity and thermal boundary layer profile. Exit length is also important in order to avoid backflow phenomena due to flow recirculation after fins array. The calculation of entrance and exit length was accomplished according to ASHRAE Standard 93-2003 [12], with equations (1) and (2) respectively.

$$
\begin{aligned}
& L_{\text {entrance }} \approx 5 \sqrt{\text { pipe length } \times \text { pipe width }} \\
& L_{\text {exit }} \approx 2.5 \sqrt{\text { pipe length } \times \text { pipe width }}
\end{aligned}
$$

Seven fin geometries where created and placed in Fin Area creating a heat sink containing two rows with four fins in each one as shown in Fig. 2, while fin height (Fh), thickness (t) and wavelength (a) are the same. Finally, in Table I. detailed dimensions of the pipe areas and fins are shown.

\section{Discontinuous \\ Wavy Fin}

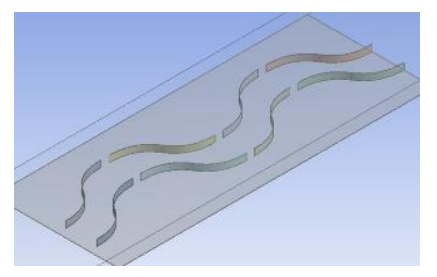

Staggered Wavy
Fin

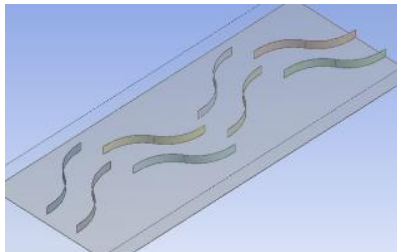

Wavy Fin $180^{\circ}$

Triangular Wavy

Fin

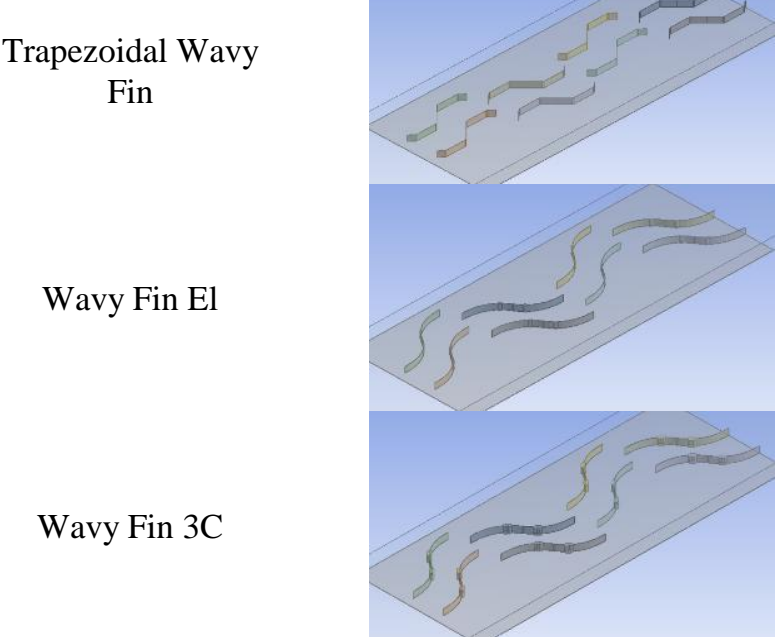

Fig. 2. Fins Geometry used in the simulations.

Table I. Dimensions of Pipe \& Fins.

\begin{tabular}{|lc|}
\hline \multicolumn{1}{|c}{ Dimension } & Size (mm) \\
\hline Pipe Height & 40 \\
Pipe Width & 400 \\
Pipe Length & 2,000 \\
Wall Thickness & 1.5 \\
Wall Width (Fin Area) & 400 \\
Wall Length (Fin Area) & 1,000 \\
Entrance Length & 500 \\
Exit Length & 500 \\
Fin Length & 225 \\
Fin Height & 20 \\
Fin Thickness & 1 \\
\hline
\end{tabular}

\section{B. Mathematical Model}

For the computational analysis, the commercial CFD code ANSYS Fluent [13] was used to predict the flow and heat transfer characteristics. The governing equations used by the CFD code to simulate each case in 3D, steady-state flow of air flowing in each proposed wavy fin passage are the Continuity Equation, the Momentum Equation and the Energy Equation.

For each fin geometry, simulations were conducted for Reynolds number in the range of $10,000<\operatorname{Re}<20,000$, that indicates the flow is turbulent. Therefore, turbulent models must be applied to get reliable results. The Realizable k- $\varepsilon$ 
turbulence model is used in all cases, adding two more equations for the CFD code to solve, the Turbulent kinetic energy $\mathrm{k}$ equation and the Turbulent energy dissipation $\mathrm{e}$ equation [14].

Realizable k- $\varepsilon$ turbulence model is high Reynolds number model, which means that is not appropriate for viscous sublayer near wall, so near-wall region should be managed by wall function. Ismail et al. [15] adopted enhanced wall function. In this paper, the scalable wall function is adopted. The governing equations are iteratively solved by the finite-volume method with Coupled pressure-velocity algorithm and discretized by the second-order upwind scheme. The convergence criterion for the residuals are the Fluent default values.

\section{Mesh \& Boundary conditions}

The computational domain is meshed with hexahedral grids using ANSYS mesh as is shown in Fig. 3(a). In order to obtain more accurate results a denser mesh grid is applied in the fin area (Fig. 3(b)), since in this area significant flow changes occur. Plus, an inflation layer was added over the walls of the duct to secure that $\mathrm{y}^{+}$values for the usage of wall functions are kept as well as for better capture of the near wall flow phenomena.

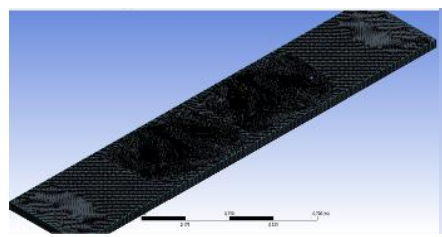

Fig. 3(a). Mesh Grid

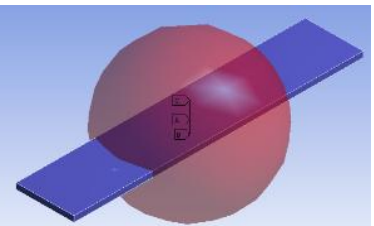

Fig. 3(b). Denser Grid Area
In the computational domain, the inlet is set as velocity inlet with different values depending by Reynolds number and a $\mathrm{T}_{\text {in }}$ set at $113 \mathrm{~K}$, outlet is set as pressure outlet and pressure is set to atmospheric. For wall boundary conditions all walls are set non-slip adiabatic except the thick wall which is set with non-slip and constant heat flux $Q=1,250 \mathrm{~W} / \mathrm{m}^{2}$ (Fig.4) [15-16] . For all simulations air properties were taken as function of temperature (Table II.) using REFPROF [17].

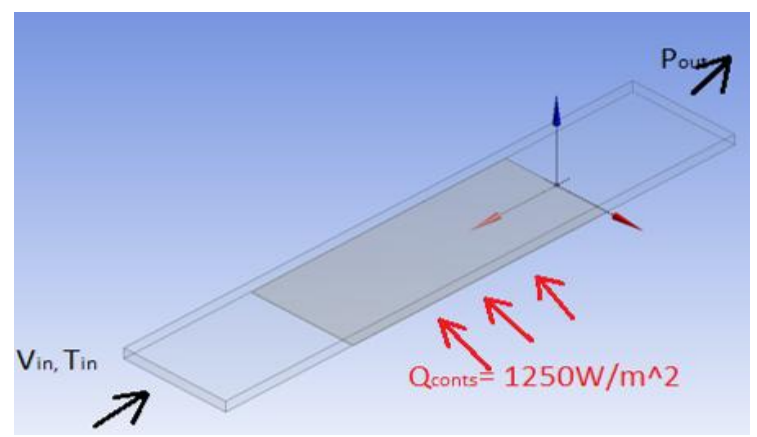

Fig 4. Boundary Conditions
Table II. Air properties at different temperature

\begin{tabular}{|c|cccc|}
\hline $\begin{array}{c}\text { Temperature } \\
(\mathbf{K})\end{array}$ & $\begin{array}{c}\text { Density } \\
\left(\mathbf{k g} / \mathbf{m}^{\mathbf{3}}\right)\end{array}$ & $\begin{array}{c}\mathbf{C}_{\mathbf{p}} \\
(\mathbf{k J} / \mathbf{k g K})\end{array}$ & $\begin{array}{c}\text { Thermal } \\
\text { conductivity } \\
(\mathbf{m W} / \mathbf{m K})\end{array}$ & $\begin{array}{c}\text { Dynamic } \\
\text { viscosity } \\
(\boldsymbol{\mu P a} \mathbf{~})\end{array}$ \\
\hline $\mathbf{1 1 3}$ & 3.1280 & 1.0264 & 10.718 & 7.9909 \\
$\mathbf{1 5 0}$ & 2.3368 & 1.0120 & 14.151 & 10.3760 \\
$\mathbf{2 0 0}$ & 1.7460 & 1.0067 & 18.502 & 13.3330 \\
$\mathbf{2 5 0}$ & 1.3948 & 1.0055 & 22.564 & 16.0380 \\
$\mathbf{3 0 0}$ & 1.1616 & 1.0063 & 26.384 & 18.5370 \\
\hline
\end{tabular}

Finally grid independence study is implemented before the numerical simulation to improve calculation accuracy with the less possible computational cost. Grid independence test results are shown in Fig. 5 for the case of $\mathrm{Re}=14,039$ for average temperature at outlet. It can be found that when the grid number reaches to $1,250,000$ cells the values of average temperature almost no longer change. Thus, for all the simulations conducted, grid density is set at almost $1,500,000$ cells.

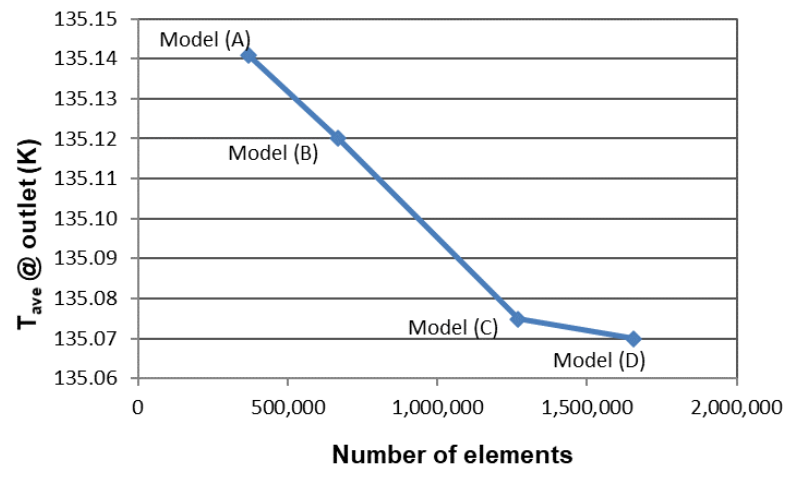

Fig 5. Grid independence study

\section{RESULTS AND DISCUSION}

\section{A. Flow field characteristics}

From Fig.6 to Fig.12 the velocity distributions for each geometry case studied are shown, on a horizontal section which is at the base of horizontal plane (X-Y plane) of the duct and fins, for Reynolds number equal to 10,049, 14,039 and 19,951 .

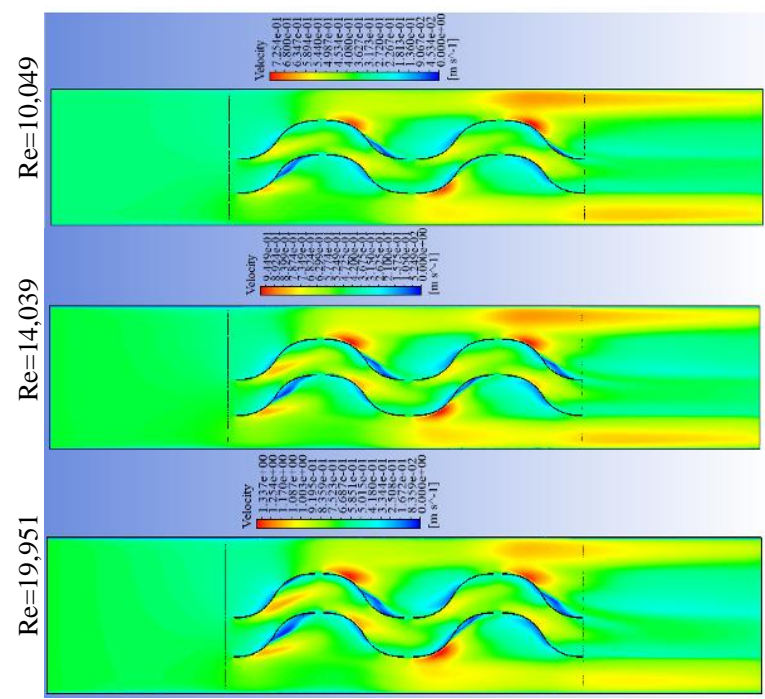

Fig 6. Discontinuous Wavy Fin-Velocity Contours 
In Fig.6, the velocity distributions for this arrangement, show some areas where it was observed that the velocity has zero value. This phenomenon occurs in the concave part of the fin and is caused by the curve geometry. At these zero-velocity regions there is the creation of vortices, enhancing the turbulence in the flow through the mixing of the fluid. That leads at better mixing of the hot air close to the fins with the cold further from the heated surface. However, the local heat transfer of these zones is reduced due to the lower velocity values which lead to neglected convection. These zones occupy more space with the increase of Reynolds number, because larger vortices are created.

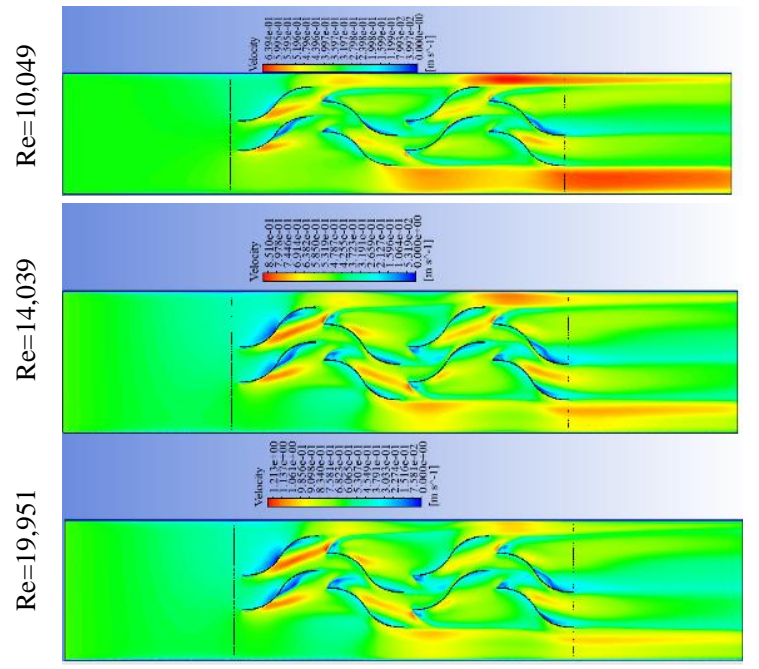

Fig.7. Staggered Wavy Fin-Velocity Contours

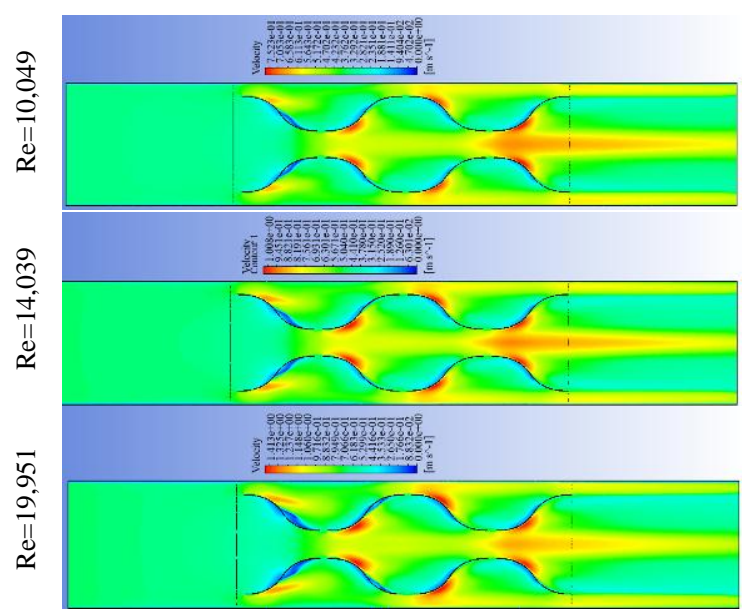

Fig.8. Wavy Fin $180^{\circ}$-Velocity Contours

In Staggered Wavy Fin (Fig.7), zero-velocity zones due to fin geometry are also observed, having the same tendency to increase when Reynolds number increases. In addition with this arrangement, better mixing of the fluid is achieved as the next fin is shifted relative to the previous one perpendicular to the flow direction. Finally, in the first sections of the fins, small zero-speed areas are observed that create vortices and boundary layer detachment. These areas are becoming more pronounced with the increase of Reynolds number.

Moving on to the arrangement where the fins have a phase difference of $180^{\circ}$ (Fig.8), the zero-velocity zones in concave fin part are also obvious. Similar to the previous cases, these areas are more intense for larger Reynolds numbers, expressing the vortices that are created. The dominant phenomenon in this case, however, is the sharp changes in speed along the fin path caused by the phase difference. Fins are forming narrower and wider passages alternately. The influence of these transitions on velocity distribution is reflected as an increase in velocity at narrow points and as a decrease at wider, as expected, according to Bernoulli's equation. The largest reductions in speed due to the increase in cross section are made at the nearest points in fin, while the core of the flow in the center has a higher speed and does not show much reduction due to inertia phenomena.

As can be seen in Fig.9, the velocity distributions for triangular wavy fin show that flow velocity is higher compared to the same arrangement consisting of classic

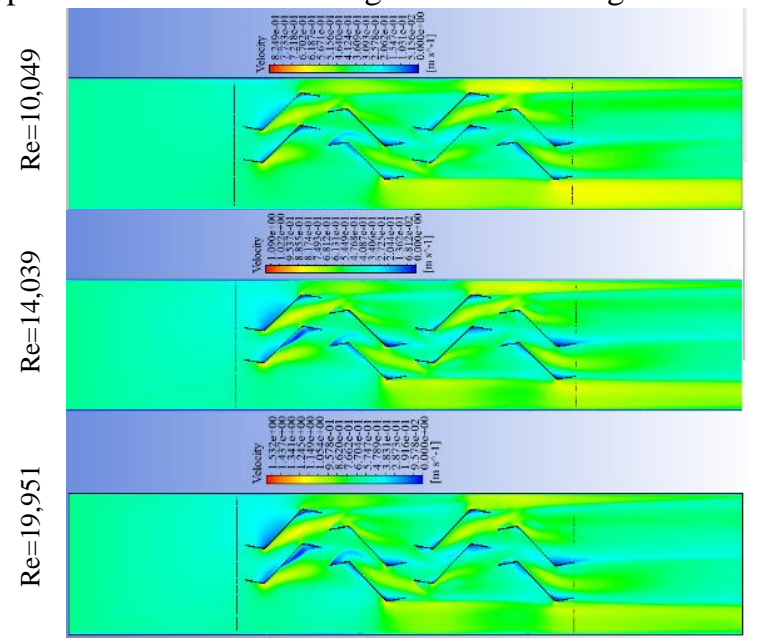

Fig.9. Triangular Wavy Fin-Velocity Contours

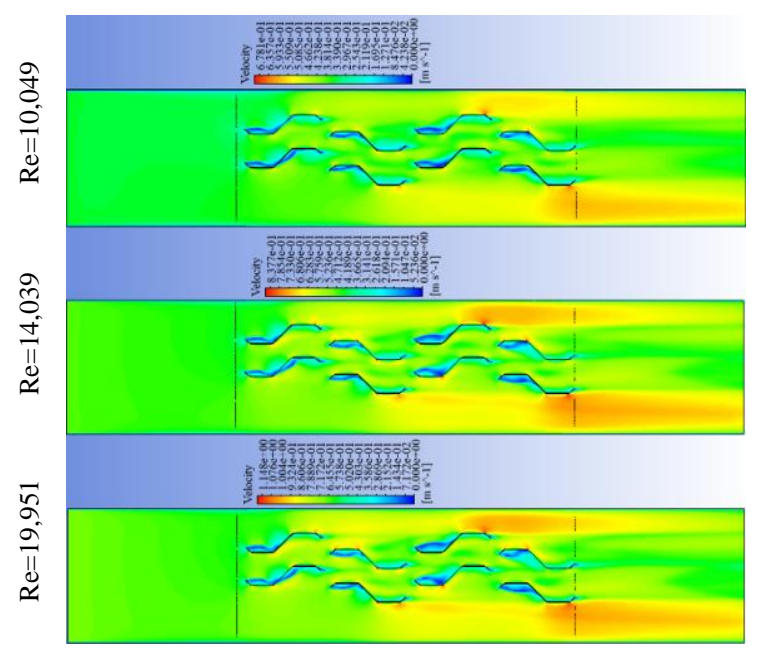

Fig.10. Trapezoidal Wavy Fin-Velocity Contours

Wavy Fin, for the same Reynolds number. Zero-velocity zones are more intense in the first row while in the next three rows they seem to be smaller, spotted at the second ventricles of Fin. Finally, the phenomenon observed at the traditional Wavy Fin of the same arrangement, in other words vortices and boundary layer separation at the beginning of each Fin, it appears in this case only in the second array, even in the highest Reynolds numbers.

Trapezoidal profile Fins in Fig.10 have the smallest velocity values compared with the rest geometries for the same Reynolds number. In addition, the zones where vortices are created and located are in the first ventricle of each Fin, occupying the first half of it. The range of these points is 
significantly larger than the other cases even for the smallest Reynolds numbers, while they increase with its increase.

Turning to the Wavy Fin with elliptical protrusions in Fig.11, their behavior is close to the same arrangement with the traditional wavy fin geometry, as their values of velocity scale are very close for corresponding Reynolds. Moreover, there are similarities between the two models in the flow of fluid in the pipeline, where they are almost identical. The only difference is spotted in the areas where vortices are created along the Fin. With the addition of elliptical protrusions, these areas become slightly more pronounced compared to the classic Fin, plus it seems the formation of some new such areas. This was a result of the addition of protrusions, which forced the fluid to move around them, causing new vortices.

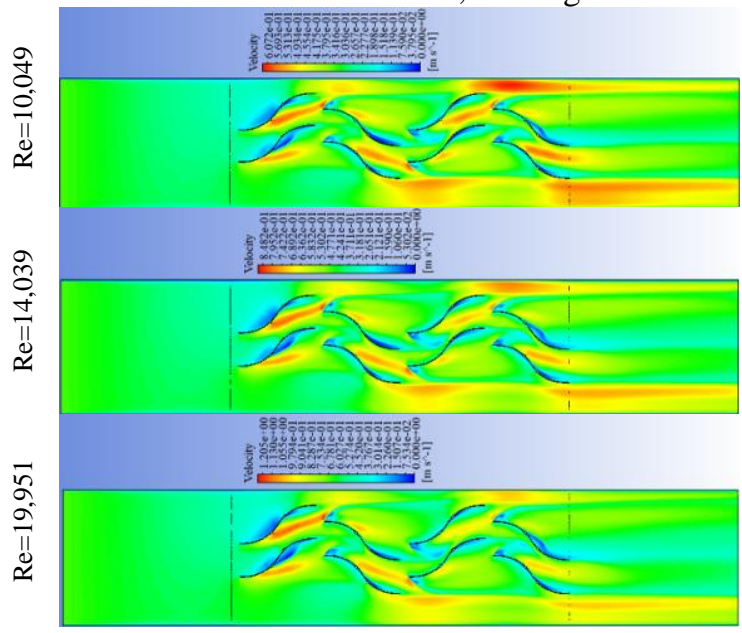

Fig.11. Wavy Fin El-Velocity Contours

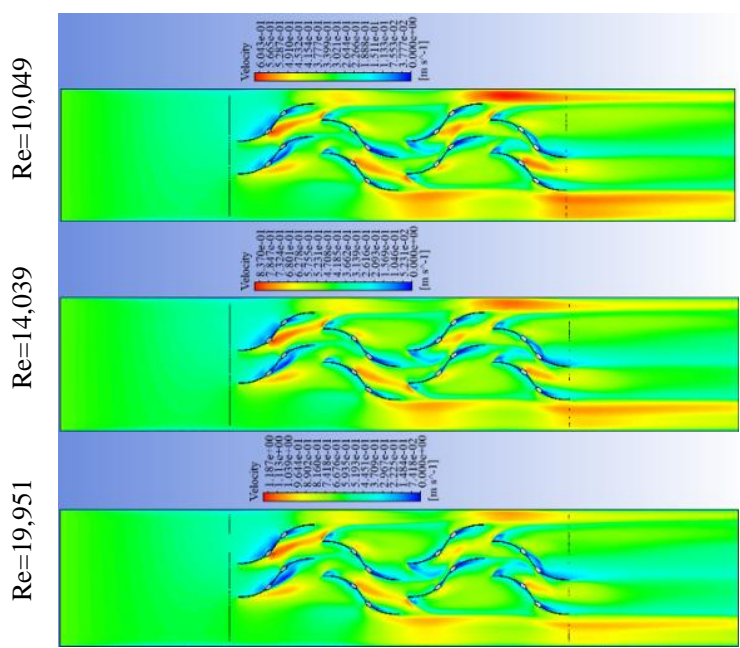

Fig.12. Wavy Fin 3C-Velocity Contours

Finally, for the model with circular shape protrusions shown in Fig.12, the velocity scale is slightly reduced compared to both the arrangement with the classic Fin and that with the elliptical protrusions. Flow characteristics are also similar to the previous two models for corresponding Reynolds numbers. Their difference is also found for this case in the zero velocity regions. It is observed that the zones of vortex creation are more intense than the previous two models due to a larger obstacle.

\section{B. Temperature distributions}

Fig. 13 to Fig.19 show the temperature distributions for each geometry case on a horizontal section which is at the base of horizontal plane (X-Y plane) of the duct and fins, same as before, at the same Reynolds numbers. For all cases the temperature of entrance length is $113(\mathrm{~K})$ up to the fin area where the heat flux is applied.

As shown in Fig.13, the temperature profile in Discontinuous Wavy Fin arrangement is disturbed by the change in the distance between the Fin and the wall as well as the array of Fin near their center. Furthermore, in the ventricles of Fin which are characterized by low velocity zones and high turbulence, the temperature is higher than the adjacent fluid that is flowing, that also disturbs the boundary layer of temperature. For the fluid flow through the Fin path, no significant disturbances in the temperature profile are observed.

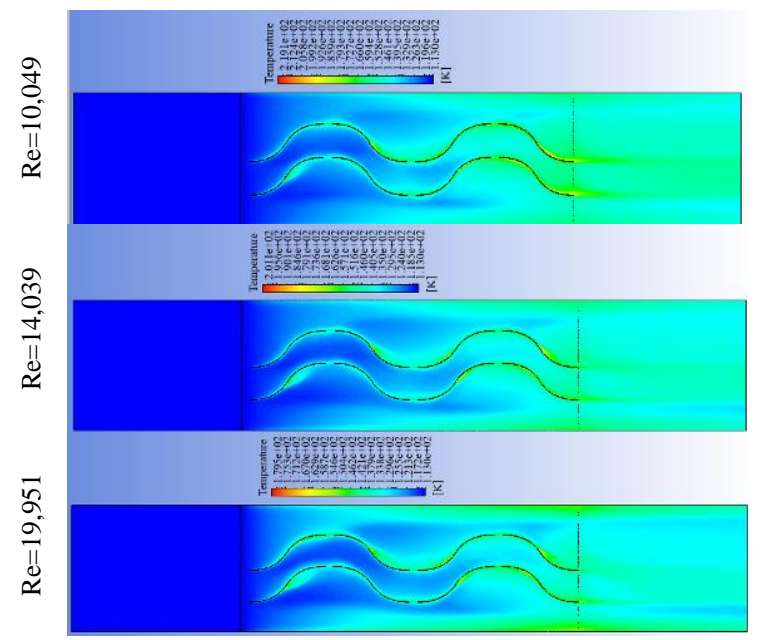

Fig.13. Discontinuous Wavy Fin-Temperature Contours

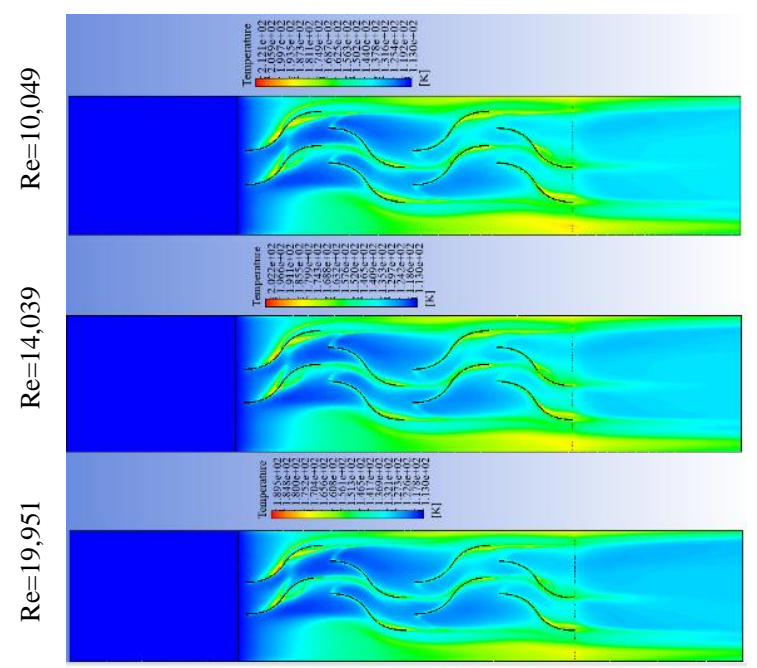

Fig.14. Staggered Wavy Fin-Temperature Contours

Staggered Wavy Fin, as shown in Fig.14, shows more intense disturbance in the temperature boundary layer of the Fin. This is due to the way the Fins are positioned, i.e. the vertical shift in the middle of the arrangement. In this way the temperature boundary layer that has been created due to fins presence is disturbed and re-formed. There is also the appearance of the concave zone phenomena. 

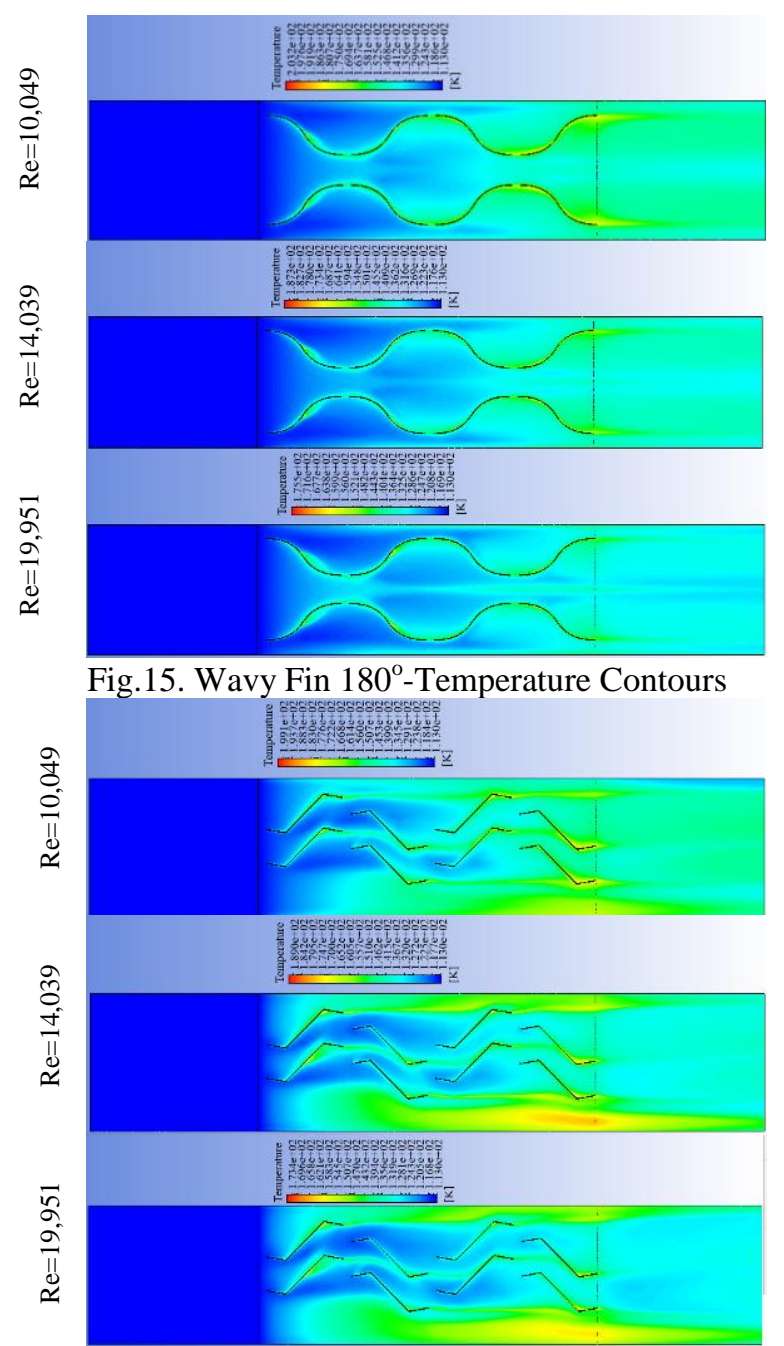

Fig.16. Triangular Wavy Fin-Temperature Contours

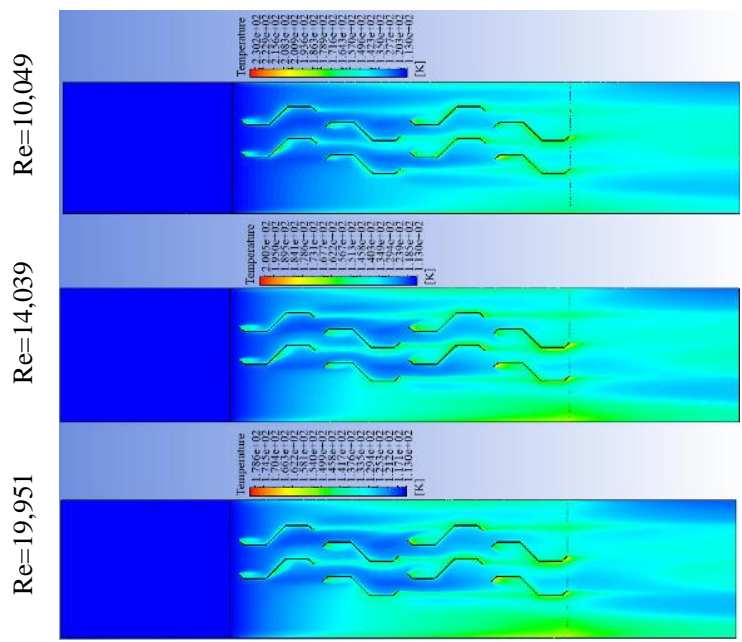

Fig.17. Trapezoidal Wavy Fin-Temperature Contours

In Wavy Fin $180^{\circ}$ in Fig.15, the changes at the temperature profile is the result of the sharp changes in the distance of Fin both between them and against the wall. The areas where the distance decreases are accompanied by a decrease in the thickness of the temperature boundary layer, while the thickness increases again as the distance increases thereafter. In addition, in concave areas the previous mentioned phenomenon still applies.

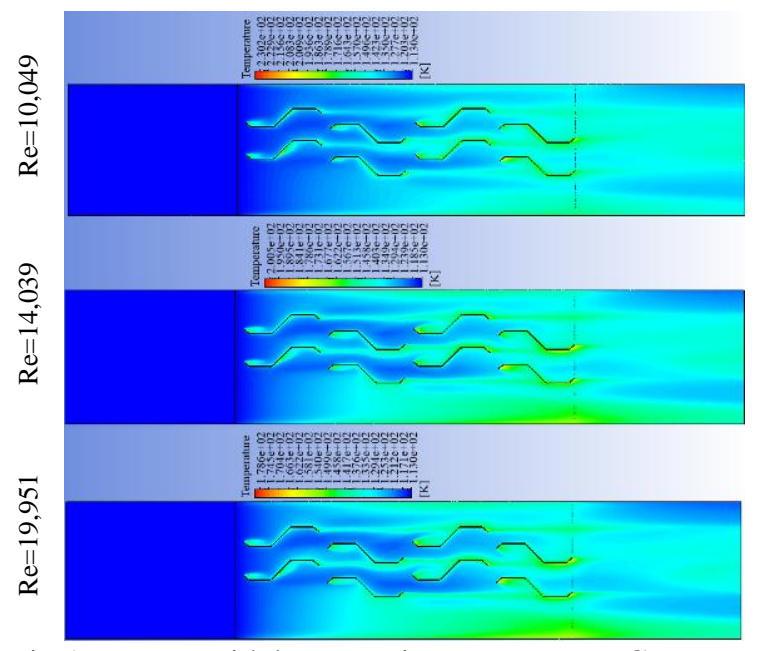

Fig.17. Trapezoidal Wavy Fin-Temperature Contours

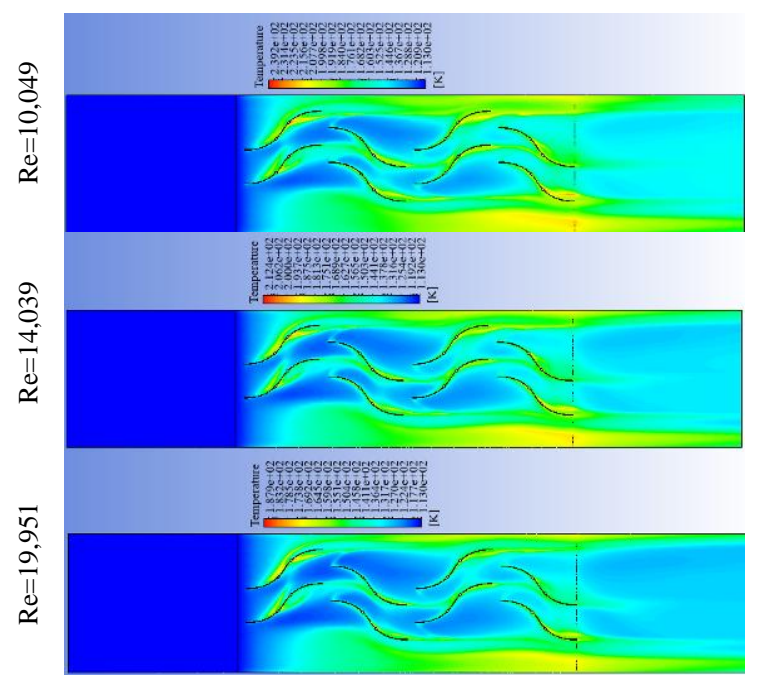

Fig.18. Wavy Fin El-Temperature Contours

From the temperature distributions of Triangular Wavy Fin (Fig.16), the boundary layer of temperature seems to be disturbed due to staggered arrangement. In this case, however, its re-growth and increase in thickness occurs after the first part of Fin, in their main trunk. In the last part of the Fin, in the zero-velocity zones, the air is observed to have higher temperatures.

In Fig. 17, Trapezoidal Wavy Fin show the highest temperature values in the areas where low speed occurred, i.e. in the first ventricles of Fin. As for the temperature profile, it is observed again that the temperature boundary layer is disturbed at the beginning of each Fin, due to the Fin's arrangement. It then maintains the same thickness for half of Fin, increasing it in its second half.

The temperature distribution of Wavy Fin El model (Fig.18) shows a similarity in temperature distribution with that of the classic Staggered Wavy Fin. However, there has been an increase in the size of areas with higher temperatures in line with the increase in low-speed points discussed in the previous subsection.

Wavy Fin 3C temperature's distribution (Fig.19) is like the classic Wavy Fin and the Wavy Fin with elliptic protrusion. Due to the larger dimples, compared to previous models, the high temperature areas which are affected by the zero-velocity zones tempt to be higher here. Finally, due to the interference of a larger obstacle, the disturbance in the temperature profile of the fluid seems more intense. 


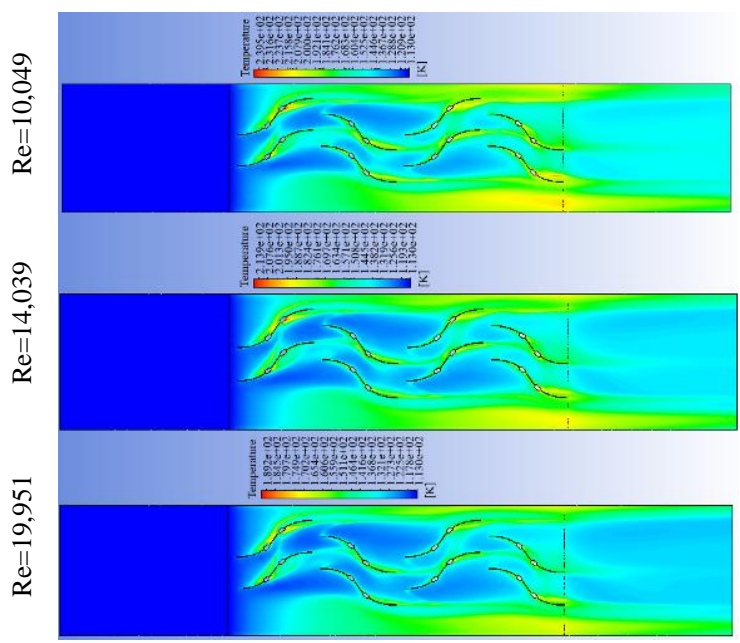

Fig.19. Wavy Fin 3C-Temperature Contours

\section{Heat Transfer Coefficient (HTC)}

Fig.20 to Fig.26 show the HTC distributions for each geometry case on a horizontal section which is at the base of horizontal plane (X-Y plane) of the duct and fins, same with the previous cases, at Reynolds numbers of 10,049, 14,039 and 19,951. For all models the entrance length and the exit length have a HTC value of $0\left(\mathrm{~W} / \mathrm{m}^{2} \mathrm{~K}\right)$ due to the fact that there is no thermal flux there. For the HTC value the Fluent variable Wall Adjacent Heat Transfer Coefficient was chosen as it is the most suitable for the problem circumstances.

Considering HTC Contour for Discontinuous Wavy Fin of Fig.20 as well as the previous distributions, it seems that the highest values of the HTC are after the areas where the pitch become minimal and increase again. Moreover, as mentioned before, in these points there is a disorder of both the temperature and velocity boundary layer, which is caused from the creation of Gortrel Vortices in the ventricles that follow, due to curvature. Thus, when the fluid passes through these points and then the boundary layers are re-created, they are accompanied by an increase in the heat transfer coefficient.

For the arrangement of Staggered Wavy Fin in Fig. 21, it can be found that the main reason for the increase in HTC is the shifted layout of Fin vertically in the flow. As can be seen from the HTC distribution, in this case the maximum values

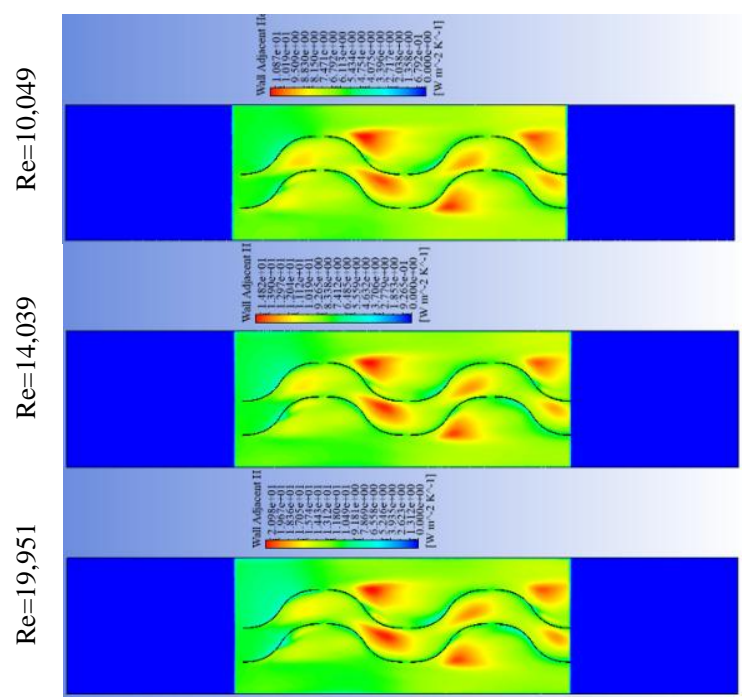

Fig.20. Discontinuous Wavy Fin-HTC Contour

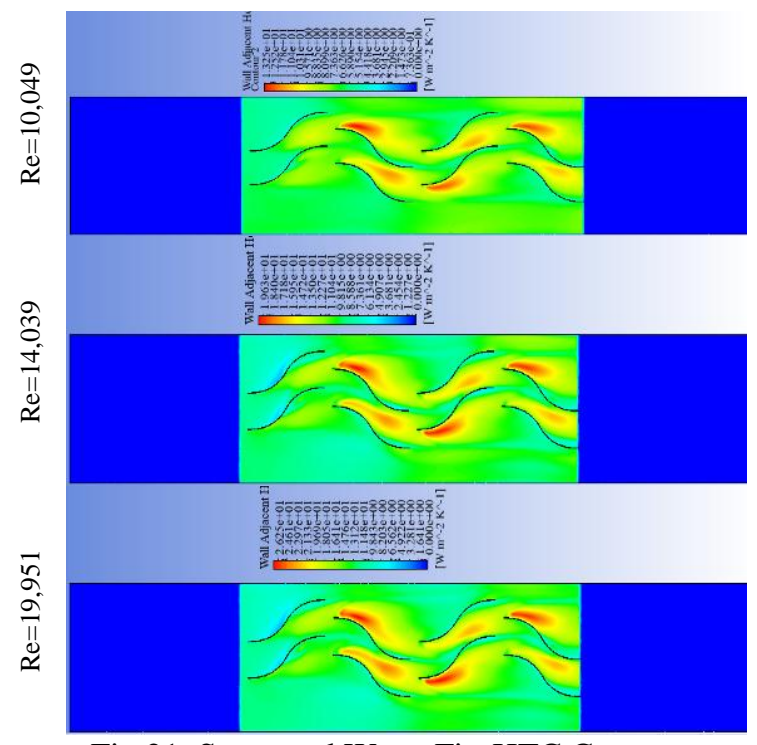

Fig.21. Staggered Wavy Fin-HTC Contour

of HTC appear at the beginning of each pair shifted relative to the previous one where the air starts to re-develop it's boundary layers. Furthermore, better mixing is achieved by this arrangement, as mentioned before at velocity distributions, which leads to heat transfer enhancement.

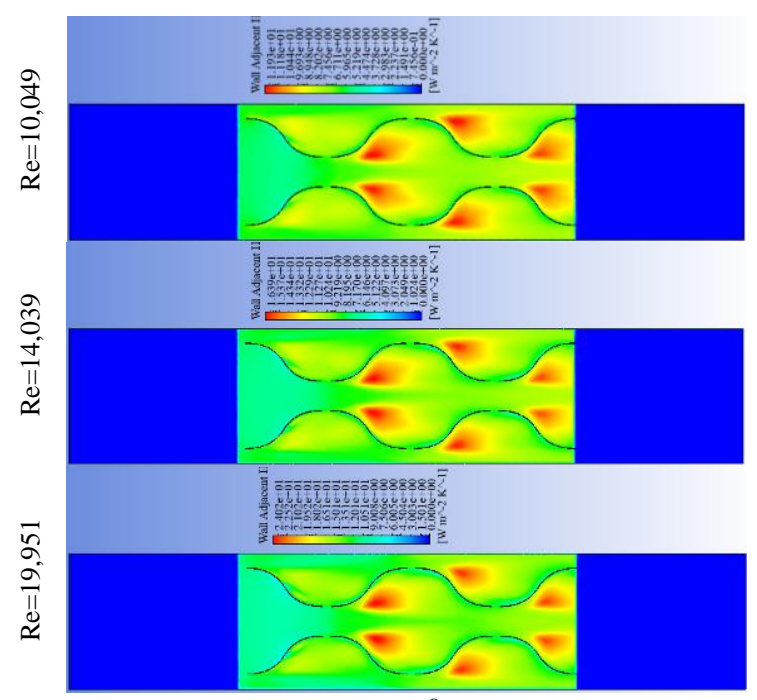

Fig.22. Wavy Fin $180^{\circ}$-HTC Contour

In Fig.22, according to HTC's distribution for Wavy Fin $180^{\circ}$, the areas with the highest values of HTC are located immediately after the narrow passages, created due to the Finn arrangement. The principle for increasing heat transfer in this case is similar to that described in the Discontinuous Wavy Fin model, with the only difference that in Wavy Fin $180^{\circ}$ there are larger changes in the distances between the Fins. This in turn leads to more intense disturbance in boundary layers, and hence a higher heat transfer coefficient. 


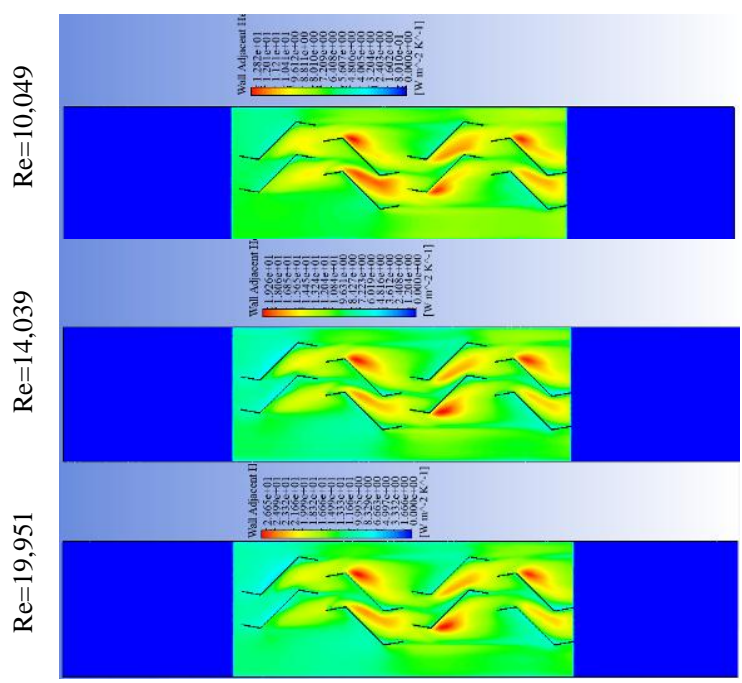

Fig.23. Triangular Wavy Fin-HTC Contour

For Triangular Fin Staggered arrangement (Fig.23) HTC contours are very close to those of the Staggered layout for the classic Wavy Fin. The mechanism that increases the heat transfer in this model is similar to that of the Staggered Wavy Fin. In this geometry however high HTC zones are in the main body of Fin after the first angle it is formed. This occurs as the air flow around the sharp corners of Fin creates disturbance at velocity and temperature boundary layers, which begin to grow again on the body of Fin. Another possible reason why HTC's high values do not appear at the

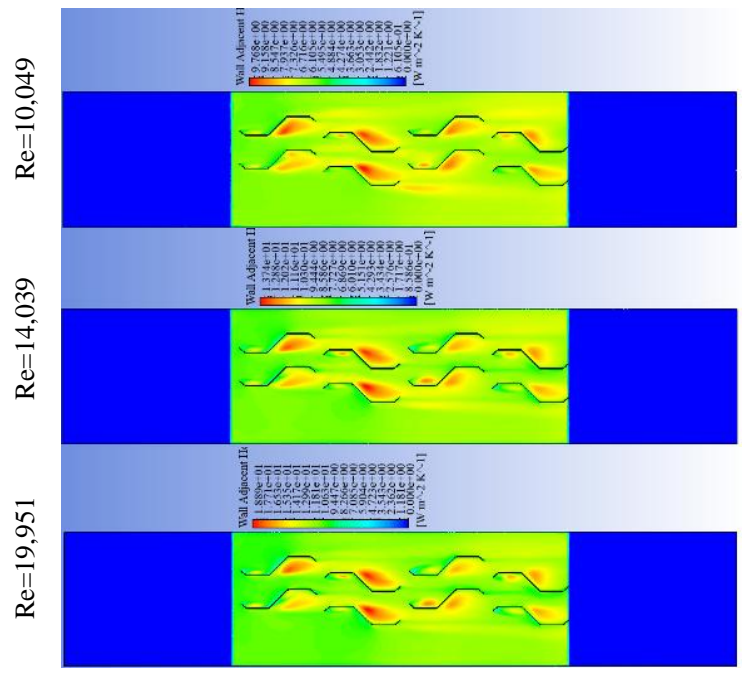

Fig.24. Trapezoidal Wavy Fin-HTC Contour

beginning of Fin is the short length of the section located in front of the corner. That means that the already disturbed by the nature of the device boundary layers do not anticipate growing because of the short length.

As shown in Fig.24, the smallest heat transfer coefficients are determined in Trapezoidal Wavy Fin model for all Reynolds numbers, comparing to any other geometry. It is observed that HTC's greatest values in this layout are in Fin's second ventricle. In addition, boundary layer separation regions, due to the staggered arrangement, are smaller in this case than those of the same layout for different Fin shapes.

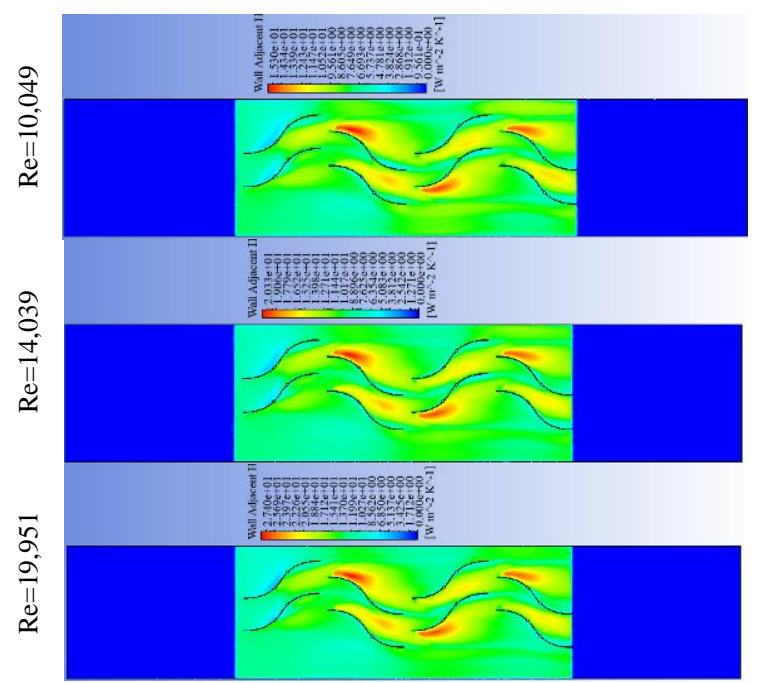

Fig.25. Wavy Fin El-HTC Contour

Wavy Fin with elliptical protrusions achieves higher HTC values as shown in Fig.25, compared to those without protrusions. From HTC's contours it can be observed a similar behavior to that of the classic Wavy Fin of the same layout. The increase HTC in this model is due to the protrusions of Fin which not only enhance the turbulence of the air but also promote its mixing. Finally, the area with the maximum values of HTC seems to be slightly smaller than that of the classic Wavy Fin.

In Fig.26 Wavy Fin 3C achieves the highest HTC values compared to the other models examined. HTC's contours look similar to those of the classic Wavy Fin model and those with elliptical additions. As in the previous case, the added

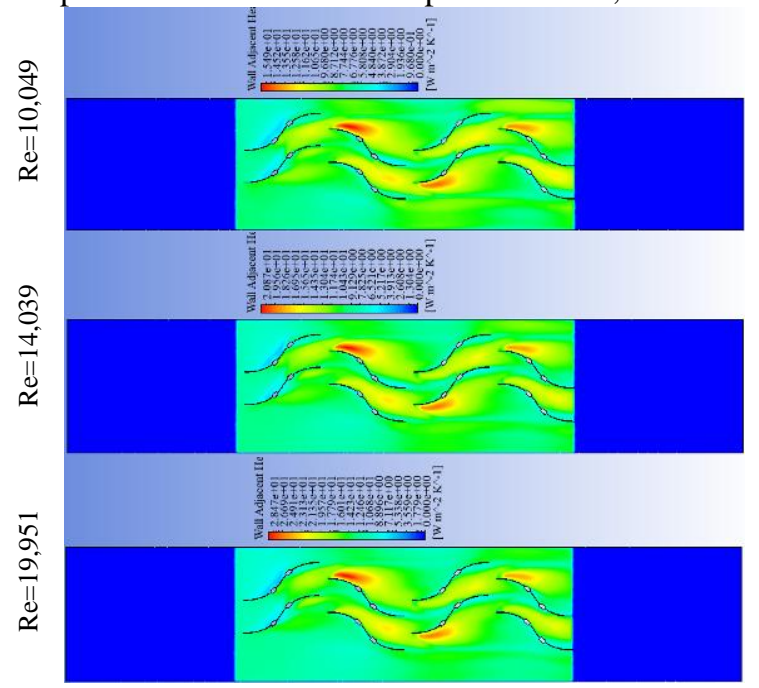

Fig.26. Wavy Fin 3C-HTC Contour

protrusions enhance the turbulence by disrupting the flow and by promoting the mixing of the air, but these phenomena are more intense than at the elliptic protrusions.

\section{Pressure drop}

Pressure drop constitutes an important parameter in, as it is directly related to the pumping power required to maintain flow. The power required to overcome flow losses is related to the operating and construction costs of the facility. Therefore, as little pressure loss as possible is desirable.

Fig. 27 shows the differences for the mean values of the total pressure, $\Delta \mathrm{P}=\mathrm{P}_{\mathrm{in}}-\mathrm{P}_{\text {out }}$, for various Reynolds numbers, 
ranging from 10,000 to 20,000 and the fin arrangements examined in the present study.

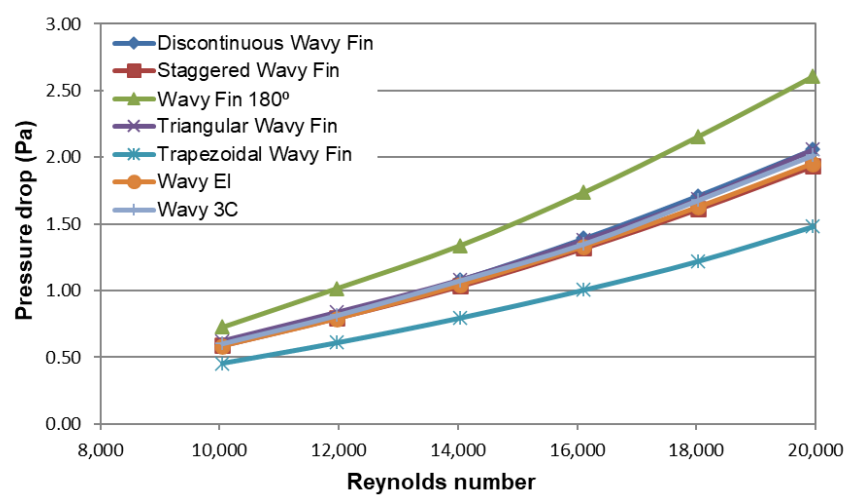

Fig.27. Pressure Drop Diagram

\section{CONCLUSION}

The main conclusion can be drawn from the present study is that the staggered arrangement performs better in terms of heat transfer, among the traditional Wavy fin geometries. The reason is that staggered arrangement causes more intense mixing and disruption of the air boundary layers. Following the $180^{\circ}$ phase shift arrangement, showing the higher pressure drop $(18,1 \%-25,73 \%)$ and lower HTC $(5.45 \%$ to $18.12 \%)$ comparing to Staggered Fins. The comparison between Discontinuous and Staggered Fins shows that HTC values differ from $18.86 \%$ to $30.63 \%$ and pressure drop from $1,1 \%$ to $6,01 \%$. For different Geometries in staggered arrangement, Triangular Fin is very close to that of the Traditional Wavy Fin for both HTC and pressure drop values $(0.52 \%-1.66 \%$ and $4.13 \%-5.94 \%$, respectively). While the comparison between Classic Wavy Fin and Trapezoidal Wavy Fin table shows that HTC values are much higher for the classic Wavy Fin (29\%-42.63\%), while the pressure drop is also higher for the classic Wavy Fin (29.45\%-32.07\%). Finally, models with protrusions show higher HTC, in particular 3.85\%-15.55\% for elliptic and $6.1 \%-16.7 \%$ for circle. As regards the pressure drop values, they are almost the same, with differences less than $1 \%$, for all the arrangements except the Wavy and the Trapezoidal Fins. Moreover, the pressure drop is reduced with the addition of cycles by $2.24 \%-4.01 \%$.

\section{REFERENCES}

[1] Tinaut, F.V., Melgar, A., \& Ali, A. A. R. "Correlations for heat transfer and flow friction characteristics of compact plate-type heat exchangers." International Journal of Heat and Mass Transfer, 1992, 35(7), pp. 1659-1665.

[2] Yousefi, M., Enayatifar, R., \& Darus, A. N. "Optimal design of plate-fin heat exchangers by a hybrid evolutionary algorithm." International Communications in Heat and Mass Transfer, 2012, 39(2), pp. 258-263.

[3] Wang,Zhe,Li, Yanzhong "Layer pattern thermal design and optimization for multistream plate-fin heat exchangers-A review" Renewable and Sustainable Energy Reviews 53, 2016, pp. 500-514

[4] S. Kakaç, A.E. Bergles, F. Mayinger, H. Yüncü, "Heat transfer enhancement of heat exchangers" Nato Asi, 1999.

[5] H. Auracher, Principles of enhanced heat transfer, Int. J. Refrig. 18 (8) 1995, pp. 565.

[6] Chao Xu, Lijun Yang, Li Li, Xiaoze Du "Experimental study on heat transfer performance improvement of wavy finned flat tube", Applied Thermal Engineering, 2015.

[7] Dong Junqi, Chen Jiangping, Chen Zhijiu, Zhou Yimin, Zhang Wenfeng "Heat transfer and pressure drop correlations for the wavy fin and flat tube heat exchangers." Applied Thermal Engineering, 2007 pp. 2066-2073.
[8] Song, Yidan Asadi, Masoud Xie, Gongnan Rocha, L. A.O. "Constructal wavy-fin channels of a compact heat exchanger with heat transfer rate maximization and pressure losses minimization." Applied Thermal Engineering 2015,Volume 75, pp. 24-32.

[9] Wen, Jian Li, Ke Wang, Chunlong Zhang, Xing Wang, Simin "Optimization investigation on configuration parameters of sine wavy fin in plate-fin heat exchanger based on fluid structure interaction analysis" International Journal of Heat and Mass Transfer, 2019, Volume 131, pp. 385-402.

[10] Xue, Yuan Ge, Zhihua Du, Xiaoze Yang, Lijun "On the heat transfer enhancement of plate fin heat exchanger." Energies , 2018, Volume 11, pp. 1-18.

[11] Nilpueng Kitti, Ahn, Ho Seon Jerng, Dong Wook Wongwises, Somchai "Heat transfer and flow characteristics of sinusoidal wavy plate fin heat sink with and without crosscut flow control." International Journal of Heat and Mass Transfer, 2019, Volume 137 pp. 565-572.

[12] Standard 93-2003 -- Methods of Testing to Determine the Thermal Performance of Solar Collectors (ANSI Approved)

[13] ANSYS® Academic Research, Release 2019 R3.

[14] Launder, B.E., Spalding, D.B. "The numerical computation of turbulent flows." Comp. Meth. Appl. Mechanics Engr., 3: pp. 269-89 1974.

[15] Ismail, L.S., Velraj, R. "Studies on fanning friction (f) and Colburn (j) factors of offset and wavy fins compact plate fin heat exchanger-a CFD approach" Numer. Heat Transf., Part A: Appl. 56 (12), 2009, pp. 987-1005.

[16] Mennad, Abed Khan, Najeeb U. Janjua, Muhammad M. Khan, Altaf H. "Computational Study of Heat Transfer Augmentation in Rectangular Channel with Upper Wall Exposed to Constant Heat Flux" Advances in Science and Engineering Technology International Conferences, ASET 2019 , pp. 1-9.

[17] Bouchenafa, R., Mohammed, H. A. "Numerical study of the thermal and hydraulic performances of heat sink made of wavy fins" Mechanics and Mechanical Engineering 2019, Volume 23, pp.150-161.

[18] REFPROF Version 10.0a.

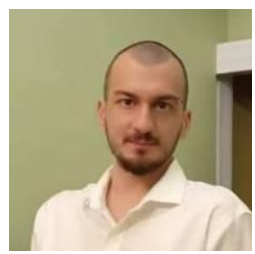

Ioannis K. Katemis, born in Athens, Greece on December $6^{\text {th }}, 1996$. He is an undergraduate student in Mechanical Engineering and Aeronautics Department at University of Patras, Greece. He has selected the division of Energy, Aeronautics \& Environment. During his studies he attended courses on Renewable Energy Systems, Multiphase flow, Fluid Mechanics, Heat and Mass Transfer, etc. His diploma thesis is parametric study for the estimation of optimum vortex generators arrangement in straight pipes.

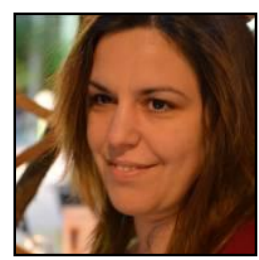

Eleni C. Douvi, born in Korinthos, Greece on March $15^{\text {th }}, 1984$. She is a post-doctoral researcher of the Mechanical Engineering and Aeronautics Department at University of Patras. Her research activities involve renewable energy, computational fluid dynamics, heat transfer and phase change materials. Her resent research activity is CFD analysis of innovative solar collector with integration of Phase Change Materials. Her previous research was the proposal of the optimum geometry of a horizontal axis tidal turbine rotor. Her doctoral thesis was experimental and computational investigation of aerodynamic behavio of wings in heavy rain, applied to horizontal axis wind turbine blades. In her diploma thesis was dealing with the experimental study of fluid mechanics applying LDA and PDA measurements. She is participating in 14 international conferences on the above scientific areas and has 10 publications on high-interested impact factor Journals. She is a member of TCG (Technical Chamber of Greece). 


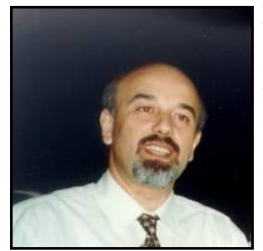

Dionissios P. Margaris, born in Zakynthos island, Greece on September $28^{\text {th }}, 1953$. He is Professor in Mechanical Engineering and Aeronautics Department at the University of Patras, Patras, Greece. His research activities/fields are multiphase flows of gas-liquid-solid particles, gas-liquid two-phase flow air-lift pump performance, centrifugal and T-junction separation modeling in gas-liquid two-phase flow, experimental and theoretical investigation of hot air dehydration of agricultural products, experimental and theoretical investigation of capillary pumped loops, steady and transient flows in pipes and network and numerical simulation of centrifugal pump performance. Also he is dealing with fluid dynamics analysis of wind turbines and aerodynamic installations, aero-acoustic analysis and environmental impacts of wind turbines. He is participating in over 130 international conferences on the above scientific areas and has over 80 publications on high-interested impact factor Journals. Prof. Dionissios P. Margaris is participating in several research projects supported by HAI, GSRT, CEC-THERMIE. Also he is member of AIAA, AHS, ASME and EUROMECH unions as well as of TCG (Technical Chamber of Greece). 